\title{
Family history of cancer in first-degree relatives and risk of gastric cancer and its precursors in a Western population
}

\author{
Minkyo Song $^{1}$ (I) $\cdot$ M. Constanza Camargo ${ }^{1} \cdot$ Stephanie J. Weinstein ${ }^{1} \cdot$ Ana F. Best $^{1} \cdot$ Satu Männistö $^{2}$. \\ Demetrius Albanes ${ }^{1}$. Charles S. Rabkin ${ }^{1}$
}

Received: 13 December 2017 / Accepted: 7 February 2018 / Published online: 17 February 2018

(c) This is a U.S. Government work and not under copyright protection in the US; foreign copyright protection may apply 2018

\begin{abstract}
Background Family history may inform risks of gastric cancer and preneoplastic lesions.

Methods We examined associations with history of cancer in first-degree relatives for 307 incident gastric cancer cases among 20,720 male smokers in a prospective study in Finland. Cox regression was used to calculate gastric cancer hazard ratios (HR) and 95\% confidence intervals (95\% CI). Logistic regression was used to estimate odds ratios (OR) and 95\% CIs for low serum pepsinogen, a marker of gastric atrophy.

Results Gastric cancer risk was associated with gastric cancer history in first-degree relatives overall (HR 1.56, 95\% CI 1.15-2.12), in fathers (HR 1.67, 95\% CI 1.09-2.55) and in siblings (HR 2.05, 95\% CI 1.25-3.38). Associations were significant for noncardia (HR 1.83, 95\% CI 1.30-2.57) but not cardia (HR 0.93, 95\% CI 0.46-1.87) cancers, and marginal for both intestinal-(HR 1.53, 95\% CI 0.92-2.55) and diffuse-type (HR 1.47, 95\% CI 0.72-3.03) histologies. Family history of other cancer types was not associated with gastric cancer risk. Family history of gastric cancer was associated with low pepsinogen (OR 1.29, 95\% CI 1.11-1.50).

Conclusions Family history of gastric cancer is strongly associated with specific subtypes of gastric cancer as well as with gastric atrophy, a risk factor for developing this malignancy.
\end{abstract}

Keywords Family history $\cdot$ Gastric cancer $\cdot$ Gastric atrophy $\cdot$ Risk factors $\cdot$ Caucasians

\section{Introduction}

Despite decreasing overall incidence over the past 50 years, particularly in developed countries, gastric cancer still ranks as the fifth most common cancer worldwide and the third leading cause of cancer mortality [1]. An estimated 952,000 new gastric cancer cases and 723,000 gastric cancer deaths

Electronic supplementary material The online version of this article (https://doi.org/10.1007/s10120-018-0807-0) contains supplementary material, which is available to authorized users.

Minkyo Song

minkyo.song@nih.gov

1 Infections and Immunoepidemiology Branch, Division of Cancer Epidemiology and Genetics, Department of Health and Human Services, National Cancer Institute, National Institutes of Health, 9609 Medical Center Drive, Bethesda, MD 20892-9776, USA

2 Department of Public Health Solutions, National Institute for Health and Welfare, Helsinki, Finland occurred globally in 2012. While age-standardized incidence rates are declining, absolute numbers of cases are increasing in some countries due to aging of the populations [2, 3]. Furthermore, divergent trends in different age, race and anatomical subgroups have been reported [4-6]. In most countries, the mortality-to-incidence ratio is more than 0.8 [7], reflecting late detection due to absence of specific symptoms and limited therapeutic options for advanced disease $[8,9]$. Therefore, identification of high risk individuals is important for surveillance and prevention of gastric cancer.

Family history is a useful screening tool for evaluating cancer risk [10, 11]. Familial aggregation may result from a combination of inherited genetic susceptibility, shared environment and common behaviors. With the exception of a few diseases with highly penetrant genes, the majority of chronic diseases result from the complex interplay of low penetrance genes with environmental and lifestyle risk factors [10]. While these polymorphisms themselves may poorly predict risk within the general population, family history has been shown to be a risk factor for many chronic diseases, 
including heart disease, osteoporosis, atopy, asthma, type 2 diabetes and certain types of cancer (e.g., colorectal, breast, and ovarian) [11].

With regard to gastric cancer, first-degree family history of gastric cancer has been consistently associated with increased risk in many populations [12]. However, most studies have been limited to retrospective case-control approaches. Only a few studies, chiefly from Asia, have examined associations with cancers diagnosed after family history was ascertained. To our knowledge, there has been no prospective analysis in a Western population of gastric cancer risks associated with family history of cancer. We, therefore, examined the association of family history of cancer with gastric cancer risk in a large prospective cohort. Furthermore, we explored whether family history of gastric cancer was associated with low serum pepsinogen as a biomarker of gastric atrophy, an intermediate step in Helicobacter pylori-related carcinogenesis.

\section{Materials and methods}

\section{Study population}

Subjects were from the alpha-tocopherol, beta-carotene (ATBC) Cancer Prevention Study, a randomized, doubleblinded primary prevention trial of daily supplementation with alpha-tocopherol, beta-carotene, or both to reduce incidence of lung or other cancers [13]. A total of 29,133 male smokers aged 50-69 who smoked for at least 5 cigarettes per day were recruited in southwestern Finland between 1985 and 1988. The intervention phase ended in April 1993, but follow-up of the participants is ongoing and for this analysis is complete through December 31st, 2014. Follow-up for cancer was ascertained using the Finnish Cancer Registry, which provides almost $100 \%$ case coverage [14].

Diagnoses of gastric cancer were classified by anatomic subsites according to the International Classification of Diseases, Ninth Revision (ICD-9) as cardia (ICD-9 code 151.0) and noncardia (ICD-9 codes 151.1-151.9, including overlapping and unspecified subsites). Histological subtypes were assessed as intestinal-type, diffuse-type and other/unspecified histologies, according to Lauren classification [15].

The study was approved by the Institutional Review Boards of both the National Cancer Institute, Bethesda, Maryland, USA and the National Public Health Institute, Helsinki, Finland. All participants provided written informed consent.

\section{Data collection}

Study participants completed questionnaires at baseline on demographic characteristics, self-reported medical history, lifestyle factors and food frequency consumption. Body mass index (BMI, $\mathrm{kg} / \mathrm{m}^{2}$ ) was calculated from height and weight measured by trained staff using standardized methods. Family history of cancer in first-degree relatives was assessed by a self-administered questionnaire that was completed in 1991, with detailed information queried for the following nine common cancers; lung, breast, prostate, bladder, pancreas, gastric, colon, rectum, and skin. After excluding those who did not complete the family history questionnaire $(n=8257)$ and those who developed cancer prior to the follow-up questionnaire $(n=156)$, non-responses comprised approximately $10-13 \%$ of answers for each cancer type, and were considered to represent no family history. Serum pepsinogens were measured for those who continued in the study for more than $3-5$ years after enrollment ( $75 \%$ of the original cohort), with low levels defined as pepsinogen I $<25 \mu \mathrm{g} / \mathrm{L}$ [16]. There were thus 19,923 participants with available data for both family history and serum pepsinogen.

\section{Statistical analyses}

Gastric cancer hazard ratios (HRs) and 95\% confidence intervals (95\% CI) associated with cancer family history were estimated using Cox proportional hazards models. For each participant, follow-up time was calculated from the date of questionnaire until the diagnosis of cancer, death or December 31st, 2014. Minimally adjusted models included age at randomization (continuous) and type of assigned intervention (alpha-tocopherol and/or beta-carotene supplements vs. respective placebos) as covariates. Full models were further adjusted for number of siblings $(0,1-3, \geq 4)$, BMI $\left(\mathrm{kg} / \mathrm{m}^{2}\right)$, pack-years of smoking (continuous), alcohol drinking (ethanol, g/day), highest level of education (categorical, high vs. low), fruit/fruit juice intake (g/day) and vegetable intake ( $\mathrm{g} /$ day). Cumulative incidence of gastric cancer in participants with or without cancer family history was calculated using the Kaplan-Meier method, with comparison between the groups performed using the log-rank test. Associations with gastric cancer family history were assessed separately for types of first-degree relationship (i.e., parent, father, mother, sibling), and for anatomic subsites (cardia and noncardia) and histologic subtypes (intestinal, diffuse and other/unspecified) of cancers. $p$ values for heterogeneity were calculated for different anatomic subsites and histologic subtypes [17]. The association between cancer family history and low serum pepsinogen was assessed by odds ratios (OR) and 95\% CI from logistic regression. A sensitivity analysis examined the effect of further adjustment by anti-H. pylori seropositivity for participants with available serology analyses for gastric cancer $(n=3382)$ and low serum pepsinogen $(n=3023)$.

Furthermore, age-specific HRs of the presence vs. absence of a family history of gastric cancer were calculated 
for participants' risk of developing gastric cancer before age 70 vs. 70 years or greater, the median age at diagnosis in this population.

We also performed a meta-analysis of previous reports on family history combined with our results. Details for the specific search strategy are included in the Supplementary Materials. The summary estimates were calculated using random-effects models. Relative risks (RRs), cumulative incidence ratios, incidence density ratios, HRs, and ORs were treated as equivalent measures for calculating effect size (ES). Adjusted RRs, when available, were preferentially included in the pooled analyses. We used Q statistics for testing heterogeneity among the different studies, and considered a significance level of $<0.1$ for rejecting the null hypothesis of homogeneity. Stratified analyses were further conducted for sex, study design, region (Asia vs. non-Asia), country, anatomic subsite, histologic subtype, and type of family relationship. The meta-analysis was conducted using the package "metan" in STATA [18].

All statistical analyses were conducted using SAS 9.3 (SAS Institute Inc, Cary, NC) and STATA/SE 14.0 for Windows (Stata Corp LP, College Station, TX). All $P$ values were two sided and were considered significant for $p<0.05$.

\section{Results}

A total of 307 incident gastric cancer cases were identified during the follow-up period. Cases were anatomically localized to 83 cardia and 224 noncardia subsites and histologically classified as 108 intestinal-type, 58 diffusetype and 141 other/unspecified. The median follow-up was 15 years for cohort participants overall. The baseline characteristics of the total cohort and gastric cancer cases are presented in Supplementary Table 1. Overall, gastric cancer patients were similar to all cohort participants in terms of age, BMI, pack-years of smoking, level of education, and daily intake of alcohol, fruit and vegetables. Gastric cancer cases had slightly more siblings than cohort subjects overall.

Associations for gastric cancer risk by family history of cancer in first-degree relatives are presented in Table 1. Except for family history of breast cancer (which was significant in the minimally adjusted model but not in the fullyadjusted model), only family history of gastric cancer was significantly associated with gastric cancer risk.

Overall, the cumulative incidence of gastric cancer was significantly higher in those with a family history of gastric cancer compared to those without (log-rank $p=0.0007)$ (Fig. 1).

Clinical characteristics of cancer cases with and without a family history of gastric cancer are shown in Table 2. Age at diagnosis, time to diagnosis, tumor grade and TNM stages did not differ significantly between the two groups.

Family history of gastric cancer in any first-degree relative was associated with 1.56 times the gastric cancer risk compared to subjects without family history of gastric cancer, after adjustment for age at randomization, type of intervention, number of siblings, BMI, pack-years of smoking, alcohol drinking, highest level of education, and fruit and vegetable intake (Table 3). Gastric cancer in one
Table 1 Risk of gastric cancer by family history of various cancers in first-degree relatives

\begin{tabular}{|c|c|c|c|c|c|c|}
\hline \multirow[t]{2}{*}{ Family history of cancer } & \multicolumn{2}{|c|}{ Case $(n=307)$} & \multicolumn{2}{|c|}{ Model 1} & \multicolumn{2}{|c|}{ Model 2} \\
\hline & $N$ & $\%$ & HR & $95 \% \mathrm{CI}$ & HR & $95 \% \mathrm{CI}$ \\
\hline Gastric & 54 & 17.6 & 1.58 & $1.18-2.13$ & 1.56 & $1.15-2.12$ \\
\hline Colon & 8 & 2.6 & 1.04 & $0.51-2.09$ & 0.80 & $0.35-1.79$ \\
\hline Rectum & 4 & 1.3 & 0.89 & $0.33-2.39$ & 0.89 & $0.33-2.40$ \\
\hline Pancreatic & 6 & 2.0 & 0.73 & $0.33-1.65$ & 0.64 & $0.26-1.54$ \\
\hline Lung & 32 & 10.4 & 1.09 & $0.76-1.57$ & 0.95 & $0.64-1.41$ \\
\hline Breast & 30 & 9.8 & 1.51 & $1.03-2.20$ & 1.36 & $0.91-2.04$ \\
\hline Prostate & 12 & 3.9 & 1.27 & $0.72-2.27$ & 1.37 & $0.77-2.44$ \\
\hline Bladder & 3 & 1.0 & 0.93 & $0.30-2.89$ & 1.00 & $0.32-3.11$ \\
\hline Skin & 12 & 3.9 & 1.31 & $0.74-2.33$ & 1.30 & $0.71-2.38$ \\
\hline Other cancers & 48 & 15.6 & 1.03 & $0.76-1.40$ & 1.02 & $0.75-1.40$ \\
\hline Any cancers ${ }^{\mathrm{a}}$ & 150 & 48.9 & 1.22 & $0.97-1.52$ & 1.17 & $0.92-1.48$ \\
\hline
\end{tabular}

Model 1: adjusted for age at randomization (years, continuous), type of assigned intervention

Model 2: adjusted for model $1+$ number of siblings $(0,1-3, \geq 4)$, BMI ( $\mathrm{kg} / \mathrm{m}^{2}$, continuous), pack-years of smoking (continuous), alcohol drinking (g/day, continuous), highest level of education (categorical), fruit intake (g/day), vegetable intake (g/day)

Number of cases and percentage shown with family history of cancer in gastric cancer incident cases

Reference is no family history of the specific cancer type

${ }^{a}$ Any cancers defined as one or more of the above listed cancers 


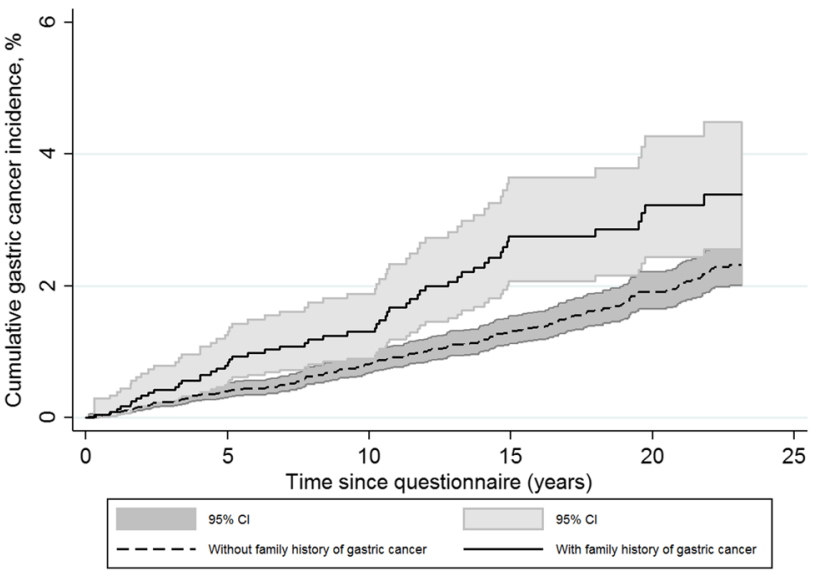

Fig. 1 Kaplan-Meier curve comparing subjects with and without a family history of gastric cancer (log-rank $p=0.0007$ )

Table 2 Clinical characteristics of incident gastric cancers by firstdegree family history of gastric cancer $(N=307)$

\begin{tabular}{|c|c|c|c|c|c|}
\hline & \multicolumn{2}{|c|}{$\begin{array}{l}\text { No fam- } \\
\text { ily history } \\
(n=253)\end{array}$} & \multicolumn{2}{|c|}{$\begin{array}{l}\text { Family his- } \\
\text { tory }(n=54)\end{array}$} & \multirow[b]{2}{*}{$p$ value } \\
\hline & $N$ & $\%$ & $N$ & $\%$ & \\
\hline \multicolumn{6}{|l|}{ Age at diagnosis in years } \\
\hline$<60$ & 5 & 2.0 & 2 & 3.7 & 0.29 \\
\hline $60-69$ & 84 & 33.2 & 17 & 31.5 & \\
\hline $70-79$ & 121 & 47.8 & 31 & 57.4 & \\
\hline$\geq 80$ & 43 & 17.0 & 4 & 7.4 & \\
\hline \multicolumn{6}{|l|}{ Time to diagnosis in years } \\
\hline$<5$ & 71 & 28.1 & 18 & 33.3 & 0.22 \\
\hline $5-10$ & 62 & 24.5 & 10 & 18.5 & \\
\hline $10-15$ & 56 & 22.1 & 21 & 38.9 & \\
\hline$\geq 15$ & 64 & 25.3 & 5 & 9.3 & \\
\hline \multicolumn{6}{|l|}{ Tumor grade } \\
\hline Well differentiated & 8 & 11.4 & 3 & 15.0 & 0.90 \\
\hline Moderately differentiated & 38 & 54.3 & 9 & 45.0 & \\
\hline Poorly differentiated & 24 & 34.3 & 8 & 40.0 & \\
\hline \multicolumn{6}{|l|}{ TNM stage at diagnosis } \\
\hline $0-\mathrm{I}$ & 28 & 31.8 & 5 & 20.8 & 0.33 \\
\hline II & 8 & 9.1 & 3 & 12.5 & \\
\hline III & 17 & 19.3 & 4 & 16.7 & \\
\hline IV & 35 & 39.8 & 12 & 50.0 & \\
\hline
\end{tabular}

Percentages for tumor grade and TNM stage at diagnosis do not include missing information

${ }^{\mathrm{a}} p$ values calculated by Mantel-Haenszel Chi Square

or both parents was associated with an HR of $1.40 \mathrm{com}-$ pared to those with no parental history of gastric cancer, although statistically insignificant. Considered separately, paternal history of gastric cancer significantly increased risk (HR 1.67), whereas maternal history was not associated with gastric cancer risk (HR 0.97). Risks associated with sibling history (HR 2.05) or both sibling and parental history of gastric cancer (HR 3.06) were somewhat greater.

First-degree family history of gastric cancer was significantly associated with noncardia gastric cancer (HR 1.83), whereas the association was null with cardia gastric cancer (HR 0.93) (Table 4). However, the difference between the two subsites did not reach statistical significance (p-heterogeneity $=0.53$ ). For Lauren classification, family history HRs of intestinal-type, diffuse-type and other/unspecified histologic types were similar but only the latter was statistically significant.

Supplementary Table 2 shows age-specific relative risk for age $<70$ vs. $\geq 70$ years. A positive family history of gastric cancer was significantly associated with elevated risk of participants' diagnosis of gastric cancer at age 70 years or less (HR 1.79), while there was no statistically significant association of family history with participants' risk after age 70 years (HR 1.11). However, the interaction between participant age and family history was not statistically significant $\left(P_{\text {interaction }}=0.19\right)$.

In the subset with available data, adjustment for anti- $H$. pylori seropositivity minimally affected the risk estimates for gastric cancer overall (Supplementary Table 3), gastric cancer by anatomic or histologic subtype (Supplementary Table 4) and low serum pepsinogen (Supplementary Table 5).

A meta-analysis of published data yielded a summary gastric cancer RR of 2.31 (95\% CI 1.99-2.68; Supplementary Figure 1) for any first-degree relative with a family history of gastric cancer, although heterogeneity across studies was statistically significant. Analyses stratified by different categories are presented in Supplementary Table 5 . The summary estimates by study design were 1.30 (95\% CI 1.26-1.34) for cohorts, and 2.56 (95\% CI 2.12-3.10) for case-control studies. By anatomical subsite, family history of gastric cancer was significantly associated with noncardia (summary RR $1.97,95 \%$ CI 1.72-2.25), but not with cardia gastric cancer (summary RR 1.46, 95\% CI 0.89-2.39). Most published studies compared presence vs. absence of gastric cancer history in parents and siblings separately. The strongest association was observed for family history of gastric cancer in siblings.

There were 1614 (8.1\%) participants with low serum pepsinogen. First-degree family history of gastric cancer was significantly associated with OR 1.29 (95\% CI 1.11-1.50). Similar to the pattern of family history associations with risk of gastric cancer, the family history associations with risk of low serum pepsinogen were stronger for sibling than parental history (Table 5). 
Table 3 Risk of gastric cancer by family history of gastric cancer in first-degree relatives

\begin{tabular}{|c|c|c|c|c|c|c|}
\hline \multirow[t]{2}{*}{ Affected family } & \multicolumn{2}{|c|}{ Case $(n=307)$} & \multicolumn{2}{|c|}{ Model 1} & \multicolumn{2}{|c|}{ Model 2} \\
\hline & $N$ & $\%$ & HR & $95 \% \mathrm{CI}$ & HR & $95 \% \mathrm{CI}$ \\
\hline \multicolumn{7}{|c|}{ Overall family history } \\
\hline Negative & 235 & 82.4 & 1.00 & & 1.00 & \\
\hline Positive & 54 & 17.6 & 1.58 & $1.18-2.13$ & 1.56 & $1.15-2.12$ \\
\hline \multicolumn{7}{|l|}{ Any parent } \\
\hline Negative & 269 & 87.6 & 1.00 & & 1.00 & \\
\hline Positive & 38 & 12.4 & 1.37 & $0.98-1.93$ & 1.40 & $0.98-1.98$ \\
\hline \multicolumn{7}{|l|}{ Father } \\
\hline Negative & 283 & 92.2 & 1.00 & & 1.00 & \\
\hline Positive & 24 & 7.8 & 1.63 & $1.07-2.47$ & 1.67 & $1.09-2.55$ \\
\hline \multicolumn{7}{|l|}{ Mother } \\
\hline Negative & 292 & 95.1 & 1.00 & & 1.00 & \\
\hline Positive & 15 & 4.9 & 1.04 & $0.62-1.75$ & 0.97 & $0.56-1.70$ \\
\hline \multicolumn{7}{|l|}{ Sibling } \\
\hline Negative & 288 & 93.8 & 1.00 & & 1.00 & \\
\hline Positive & 19 & 6.2 & 2.22 & $1.39-3.54$ & 2.05 & $1.25-3.38$ \\
\hline \multicolumn{7}{|l|}{ Parent and sibling } \\
\hline Neither & 254 & 82.7 & 1.00 & & 1.00 & \\
\hline Either & 49 & 16.0 & 1.51 & $1.11-2.05$ & 1.47 & $1.07-2.03$ \\
\hline Both & 4 & 1.3 & 2.92 & $1.09-7.86$ & 3.06 & $1.13-8.24$ \\
\hline
\end{tabular}

Model 1: adjusted for age at randomization (years, continuous), type of assigned intervention

Model 2: adjusted for model $1+$ number of siblings $(0,1-3, \geq 4)$, BMI ( $\mathrm{kg} / \mathrm{m}^{2}$, continuous), pack-years of smoking (continuous), alcohol drinking ( $\mathrm{g} /$ day, continuous), highest level of education (categorical), fruit intake (g/day), vegetable intake (g/day)

\begin{tabular}{|c|c|c|c|c|c|c|}
\hline & \multicolumn{2}{|c|}{ Case $(n=307)$} & \multicolumn{2}{|c|}{ Model 1} & \multicolumn{2}{|c|}{ Model 2} \\
\hline & $N$ & $\%$ & HR & $95 \% \mathrm{CI}$ & HR & $95 \% \mathrm{CI}$ \\
\hline \multicolumn{7}{|l|}{ Anatomic subsite } \\
\hline Cardia $(n=83)$ & 10 & 12.1 & 1.03 & $0.52-1.96$ & 0.93 & $0.46-1.87$ \\
\hline Noncardia $(n=224)$ & 44 & 19.6 & 1.82 & $1.31-2.53$ & 1.83 & $1.30-2.58$ \\
\hline p-Heterogeneity & & & 0.62 & & 0.53 & \\
\hline \multicolumn{7}{|l|}{ Lauren classification } \\
\hline Intestinal $(n=108)$ & 19 & 17.6 & 1.56 & $0.95-2.56$ & 1.53 & $0.92-2.55$ \\
\hline Diffuse $(n=58)$ & 10 & 17.2 & 1.58 & $0.80-3.13$ & 1.47 & $0.72-3.03$ \\
\hline Other/unspecified $(n=141)$ & 25 & 17.7 & 1.61 & $1.04-2.48$ & 1.61 & $1.02-2.53$ \\
\hline p-Heterogeneity ${ }^{\mathrm{a}}$ & & & 0.54 & & 0.68 & \\
\hline
\end{tabular}

Model 1: adjusted for age at randomization (years, continuous), type of assigned intervention

Model 2: adjusted for model $1+$ number of siblings $(0,1-3, \geq 4)$, BMI ( $\mathrm{kg} / \mathrm{m}^{2}$, continuous), pack-years of smoking (continuous), alcohol drinking ( $\mathrm{g} /$ day, continuous), highest level of education (categorical), fruit intake (g/day), vegetable intake ( $\mathrm{g} /$ day)

${ }^{a}$ p-Heterogeneity calculated for intestinal vs. diffuse-type gastric cancers

\section{Discussion}

In this large prospective study with a long follow-up period, first-degree family history of gastric cancer was associated with risk of gastric cancer. In particular, having a father or a sibling with gastric cancer significantly increased the risk. Furthermore, family history of gastric cancer was associated with low pepsinogen, a biomarker of gastric atrophy, which is a known precursor for gastric cancer. Family history of other cancer types was not associated with gastric cancer risk. 
Table 5 Association of low serum pepsinogen with firstdegree family history of gastric cancer

\begin{tabular}{|c|c|c|c|c|c|c|}
\hline \multirow[t]{2}{*}{ Affected family } & \multicolumn{2}{|c|}{ Low pepsinogen } & \multicolumn{2}{|c|}{ Model 1} & \multicolumn{2}{|c|}{ Model 2} \\
\hline & $N$ & $\%$ & OR & $95 \% \mathrm{CI}$ & OR & $95 \% \mathrm{CI}$ \\
\hline \multicolumn{7}{|c|}{ Overall family history } \\
\hline Negative & 1371 & 84.9 & 1.00 & & 1.00 & \\
\hline Positive & 243 & 15.1 & 1.29 & $1.11-1.49$ & 1.29 & $1.11-1.50$ \\
\hline \multicolumn{7}{|l|}{ Any parent } \\
\hline Negative & 1445 & 89.5 & 1.00 & & 1.00 & \\
\hline Positive & 169 & 10.5 & 1.16 & $0.98-1.38$ & 1.21 & $1.02-1.44$ \\
\hline \multicolumn{7}{|l|}{ Father } \\
\hline Negative & 1523 & 94.4 & 1.00 & & 1.00 & \\
\hline Positive & 91 & 5.6 & 1.17 & $0.94-1.47$ & 1.19 & $0.95-1.50$ \\
\hline \multicolumn{7}{|l|}{ Mother } \\
\hline Negative & 1531 & 94.9 & 1.00 & & 1.00 & \\
\hline Positive & 83 & 5.1 & 1.13 & $0.90-1.43$ & 1.20 & $0.95-1.52$ \\
\hline \multicolumn{7}{|l|}{ Sibling } \\
\hline Negative & 1538 & 95.3 & 1.00 & & 1.00 & \\
\hline Positive & 76 & 4.7 & 1.44 & $1.13-1.85$ & 1.31 & $1.01-1.72$ \\
\hline \multicolumn{7}{|c|}{ Parent and sibling } \\
\hline Neither & 1378 & 85.4 & 1.00 & & 1.00 & \\
\hline Either & 227 & 14.1 & 1.26 & $1.09-1.47$ & 1.28 & $1.09-1.49$ \\
\hline Both & 9 & 0.6 & 1.19 & $0.59-2.38$ & 1.05 & $0.50-2.19$ \\
\hline
\end{tabular}

Model 1: adjusted for age at randomization (years, continuous), type of assigned intervention

Model 2: adjusted for model $1+$ number of siblings $(0,1-3, \geq 4)$, BMI ( $\mathrm{kg} / \mathrm{m}^{2}$, continuous), pack-years of smoking (continuous), alcohol drinking (g/day, continuous), highest level of education (categorical), fruit intake (g/day), vegetable intake (g/day)

Low pepsinogen defined as serum pepsinogen $\mathrm{I}<25 \mu \mathrm{g} / \mathrm{L}$
Family history of gastric cancer has long been considered a risk factor for gastric cancer. However, most prior evidence is from case-control studies, which may be prone to exaggeration of effect size due to recall bias. Indeed, our meta-analyses found a much stronger summary association in case-control studies (2.6-fold) than cohort studies (1.3fold). A limited number of prospective cohort studies have been conducted, all in Asian populations, with inconsistent results [19-21]. One study in Korea based on the National Health Insurance Corporation data developed a prediction model incorporating family history of gastric cancer [19]. The HR for family history was 1.30 (95\% CI 1.25-1.35) in males, and 1.27 (95\% CI 1.19-1.37) in females. In another prediction model in a Japanese population, the HR was 1.37 (95\% CI 0.98-1.90) [20]. Another Japanese study reported an OR of 0.89 (95\% CI 0.40-1.97) for males and 1.73 (95\% CI 0.82-3.65) in females in a nested case-control design [21]. To our knowledge, our study is the first prospective cohort study to evaluate the association of family history of gastric cancer in a Western population. Our risk estimate was in line with the Asian cohort studies, but smaller than most of the case-control studies.

Given that family history reflects not only genetic association but also shared environment and common behaviors, it is noteworthy that family history of other cancer types that share the same risk factors with gastric cancer, such as processed meat consumption in colorectal cancer [22], was not associated with gastric cancer risk. Interestingly, we observed a significant association with breast cancer family history in a minimally adjusted model, although the significance was attenuated after adjusting for additional covariates. Breast cancer is the second most frequent cancer type in $\mathrm{CDH} 1$-affected families, a type of hereditary diffuse gastric cancer [23, 24]. In fact, one US study reported that family history of breast cancer was associated with a 1.8fold increased OR (95\% CI 1.1-2.8) of noncardia gastric cancer [25]. No other specific cancer types were reported to be associated with gastric cancer risk in observational studies [25-34]. Conversely, one study had reported family history of gastric cancer to be associated with breast cancer risk [35]. The potential familial associations of breast and gastric cancers need further elucidation.

No previous prospective cohort studies have investigated risk differences by type of first-degree relationships. We found a higher association with sibling than parental family history in our cohort data as well as our meta-analysis of prior case-control studies [27, 28, 31, 32, 34, 36, 37]. Greater risk with sibling history may be due to the additional 
contribution of shared environment and similar lifestyle habits from childhood to adolescence, since $H$. pylori infection is generally acquired in childhood [38]. We also found a stronger association with paternal family history compared to maternal family history, which is inconsistent with most previous studies [28, 29, 31, 32, 39]. The discrepancy of our study may be due to limited sample size and needs further investigation.

To our knowledge, this is the first prospective study to investigate family history risk for subgroups of gastric cancer. In most case-control studies, the family history association was insignificant in cardia cancer and significant with noncardia cancer [25, 32, 40], and even one study which reported both subsites significant showed a stronger association with noncardia cancer [41]. Our study found a significant association in noncardia cancer only, although this association was not statistically different from cardia cancer. With regard to Lauren classification, previous studies reported inconsistent results, overall rendering a similar magnitude of association in our meta-analysis. We report marginally elevated risk for both intestinal and diffuse-type cancers, with no statistically significant difference between the two types.

We did not observe a significant interaction for agespecific relative risks. Family history of gastric cancer in fathers, and/or siblings (but not in mothers) was associated with a statistically significant increased risk among individuals younger than 70 years. Previous studies of age-specific risks have been inconsistent $[31,34,39]$. Our study population had a median age at diagnosis at 70 , which may have diluted hypothetically strong genetic association of early onset cancer.

Serum pepsinogen, which is reflective of mucosal atrophy of the stomach $[42,43]$, is a useful predictor for gastric cancer risk [44]. In a study restricted to $H$. pylori-infected individuals in Italy, serum pepsinogen levels were lower in first-degree relatives of gastric cancer patients when compared to age- and sex-matched dyspeptic patients with no family history [45]. However, a study from Germany found no association of gastric cancer family history with development of chronic atrophic gastritis, defined by serum pepsinogen status, after a five-year follow-up [46]. A Japanese study, based on endoscopically diagnosed gastric atrophy rather than serology, found an association with family history of gastric cancer (OR 3.97, 95\% CI 1.41-10.60) [47]. Our finding that family history of gastric cancer is associated with this early stage of the gastric carcinogenesis cascade may partially explain the family history association with gastric cancer.

We found risks of both gastric cancer and low serum pepsinogen more strongly associated with sibling than parental history of gastric cancer. A potential explanation is concordance of $H$. pylori infection status as well as molecular subtype, which tend to be stronger between siblings than between parents and offspring [48-51].

Our study has several strengths. First, the prospective design avoids selection or recall biases and reverse causation. Second, the number of cases in our study was large enough to allow a more comprehensive analysis across age, anatomical and histological subgroups, as well as accounting for potential confounders. Third, we observed an association of family history with low pepsinogen supporting the hypothesized pathophysiologic pathway.

One of the limitations of our study is that the findings from our study population, who are male smokers, may have limited generalizability. Nonetheless, risk estimates from the current analysis were consistent with previous reports. Another limitation may be the method of assessment of our main exposure variable. Family history was based on self-report from questionnaires, and like most studies, it was infeasible to validate the reported cancers. However, the $18 \%$ prevalence of family history among our cases was midway between the lowest (11\% in a US population [25]) and highest (21\% in an Italian study [37]) Western populations included in the meta-analysis. Underreporting may be possible but is unlikely to differ by cancer status, since the information was collected years prior to diagnosis [52]. Nondifferential misclassification usually attenuates risk estimates toward the null, with expected true estimates larger than the observed values [53]. Moreover, since the information regarding family history was only collected once during the study, it is possible that additional family members were diagnosed with gastric cancer during the follow-up period, which would have led to underestimating the prevalence of cancer family history. Detection bias may result from more attentive screening of those with a family history of cancer, but mass screening for gastric cancer is not implemented in Finland. In fact, in our study population neither age at diagnosis nor stage at diagnosis differed significantly between cases with and without family history. Furthermore, we did not have information about inheritable syndromes associated with gastric cancer risk, such as hereditary diffuse gastric cancer, gastric adenocarcinoma and proximal polyposis of the stomach, familial intestinal gastric cancer, hereditary nonpolyposis colon cancer, Li-Fraumeni syndrome, Peutz-Jeghers syndrome and familial adenomatous polyposis $[54,55]$. However, hereditary cancer syndromes are only linked to less than $3 \%$ of gastric cancer cases [56].

In summary, our long-term prospective study provides additional evidence that a family history of gastric cancer conveys a substantially increased risk for gastric cancer. Mass screening for gastric cancer is impractical in low and moderate incidence populations. Nevertheless, our results suggest that risk stratification by the simple assessment of family history may identify a higher risk subgroup that 
warrants targeting consideration for targeted screening approaches.

Acknowledgements This study was supported by the Intramural Research Program, Division of Cancer Epidemiology and Genetics, National Cancer Institute, National Institutes of Health, Department of Health and Human Services. The ATBC Study was supported by funding provided by the Intramural Research Program of the National Cancer Institute and US Public Health Service contracts [HHSN261201500005C].

\section{Compliance with ethical standards}

Human rights statement and informed consent All procedures were in accordance with the ethical standards of the responsible committee on human experimentation and with the Helsinki Declaration of 1964 and later versions. The study was approved by the Institutional Review Boards of both the National Cancer Institute, Bethesda, Maryland, USA and the National Public Health Institute, Helsinki, Finland. All participants provided written informed consent.

Conflict of interest The authors declare no conflict of interest.

\section{References}

1. Ferlay J, et al. Cancer incidence and mortality worldwide: sources, methods and major patterns in GLOBOCAN 2012. Int J Cancer. 2015;136(5):E359-86.

2. Inoue M, Tsugane S. Epidemiology of gastric cancer in Japan. Postgrad Med J. 2005;81(957):419-24.

3. Song M, Lee H, Kang D. Epidemiology and screening of gastric cancer in Korea. J Korean Med Assoc. 2015;58(3):183-90.

4. Camargo MC, et al. Divergent trends for gastric cancer incidence by anatomical subsite in US adults. Gut. 2011;60(12):1644-9.

5. Botterweck AA, et al. Trends in incidence of adenocarcinoma of the oesophagus and gastric cardia in ten European countries. Int J Epidemiol. 2000;29(4):645-54.

6. Song M, et al. Age and sex interactions in gastric cancer incidence and mortality trends in Korea. Gastric Cancer. 2015;18(3):580-9.

7. Shen L, et al. Management of gastric cancer in Asia: resourcestratified guidelines. Lancet Oncol. 2013;14(12):e535-47.

8. Orditura M, et al. Treatment of gastric cancer. World J Gastroenterol. 2014;20(7):1635-49.

9. Rosati G, Ferrara D, Manzione L. New perspectives in the treatment of advanced or metastatic gastric cancer. World J Gastroenterol. 2009;15(22):2689-92.

10. Yoon PW, et al. Can family history be used as a tool for public health and preventive medicine? Genet Med. 2002;4(4):304-10.

11. Guttmacher AE, Collins FS, Carmona RH. The family historymore important than ever. N Engl J Med. 2004;351(22):2333-6.

12. Yaghoobi M, Bijarchi R, Narod SA. Family history and the risk of gastric cancer. Br J Cancer. 2010;102(2):237-42.

13. The ATBC Cancer Prevention Study Group. The alpha-tocopherol, beta-carotene lung cancer prevention study: design, methods, participant characteristics, and compliance. Ann Epidemiol. 1994;4(1):1-10.

14. Korhonen P, et al. The Finnish Cancer Registry as follow-up source of a large trial cohort-accuracy and delay. Acta Oncol. 2002;41(4):381-8.

15. Lauren $P$. The two histological main types of gastric carcinoma: diffuse and so-called intestinal-type carcinoma. An attempt at a histo-clinical classification. Acta Pathol Microbiol Scand. 1965;64:31-49.

16. Varis K, et al. Implications of serum pepsinogen I in early endoscopic diagnosis of gastric cancer and dysplasia. Helsinki Gastritis Study Group. Scand J Gastroenterol. 2000;35(9):950-6.

17. Wang M, et al. Statistical methods for studying disease subtype heterogeneity. Stat Med. 2016;35(5):782-800.

18. Higgins JP, et al. Measuring inconsistency in meta-analyses. BMJ. 2003;327(7414):557-60.

19. Eom BW, et al. Prediction model for gastric cancer incidence in Korean population. PLoS ONE. 2015;10(7):e0132613.

20. Charvat $\mathrm{H}$, et al. Prediction of the 10 -year probability of gastric cancer occurrence in the Japanese population: the JPHC study cohort II. Int J Cancer. 2016;138(2):320-31.

21. Yatsuya $\mathrm{H}$, et al. Individual and joint impact of family history and Helicobacter pylori infection on the risk of stomach cancer: a nested case-control study. Br J Cancer. 2004;91(5):929-34.

22. Vieira AR, et al. Foods and beverages and colorectal cancer risk: a systematic review and meta-analysis of cohort studies, an update of the evidence of the WCRF-AICR continuous update project. Ann Oncol. 2017;28(8):1788-802.

23. Fitzgerald RC, et al. Hereditary diffuse gastric cancer: updated consensus guidelines for clinical management and directions for future research. J Med Genet. 2010;47(7):436-44.

24. Pharoah PD, et al. Incidence of gastric cancer and breast cancer in CDH1 (E-cadherin) mutation carriers from hereditary diffuse gastric cancer families. Gastroenterology. 2001;121(6):1348-53.

25. Dhillon PK, et al. Family history of cancer and risk of esophageal and gastric cancers in the United States. Int J Cancer. 2001;93(1):148-52.

26. Safaee A, et al. Family history of cancer and risk of gastric cancer in Iran. Asian Pac J Cancer Prev. 2011;12(11):3117-20.

27. Foschi R, et al. Family history of cancer and stomach cancer risk. Int J Cancer. 2008;123(6):1429-32.

28. Eto K, et al. Familial clustering in subgroups of gastric cancer stratified by histology, age group and location. Eur J Surg Oncol. 2006;32(7):743-8.

29. Bakir T, et al. Gastric cancer and other organ cancer history in the parents of patients with gastric cancer. Eur J Cancer Prev. 2003;12(3):183-9.

30. Minami Y, Tateno H. Associations between cigarette smoking and the risk of four leading cancers in Miyagi Prefecture, Japan: a multi-site case-control study. Cancer Sci. 2003;94(6):540-7.

31. Lissowska $\mathbf{J}$, et al. Family history and risk of stomach cancer in Warsaw, Poland. Eur J Cancer Prev. 1999;8(3):223-7.

32. Inoue $\mathbf{M}$, et al. Family history and subsite of gastric cancer: data from a case-referent study in Japan. Int J Cancer. 1998;76(6):801-5.

33. Gajalakshmi CK, Shanta V. Lifestyle and risk of stomach cancer: a hospital-based case-control study. Int J Epidemiol. 1996;25(6):1146-53.

34. La Vecchia C, et al. Family history and the risk of stomach and colorectal cancer. Cancer. 1992;70(1):50-5.

35. Turati F, et al. Family history of cancer and the risk of cancer: a network of case-control studies. Ann Oncol. 2013;24(10):2651-6.

36. Huang $X$, et al. Effect of life styles on the risk of subsite-specific gastric cancer in those with and without family history. J Epidemiol. 1999;9(1):40-5.

37. Palli D, et al. Family history and risk of stomach cancer in Italy. Cancer Epidemiol Biomarkers Prev. 1994;3(1):15-8.

38. Weyermann M, Rothenbacher D, Brenner H. Acquisition of Helicobacter pylori infection in early childhood: independent contributions of infected mothers, fathers, and siblings. Am J Gastroenterol. 2009;104(1):182-9.

39. Shin CM, et al. Stomach cancer risk in gastric cancer relatives: interaction between Helicobacter pylori infection and family 
history of gastric cancer for the risk of stomach cancer. J Clin Gastroenterol. 2010;44(2):e34-9.

40. Garcia-Gonzalez MA, et al. Gastric cancer susceptibility is not linked to pro-and anti-inflammatory cytokine gene polymorphisms in whites: a nationwide multicenter study in Spain. Am J Gastroenterol. 2007;102(9):1878-92.

41. Gao Y, et al. Family history of cancer and risk for esophageal and gastric cancer in Shanxi, China. BMC Cancer. 2009;9:269.

42. Samloff IM. Immunologic studies of human group I pepsinogens. J Immunol. 1971;106(4):962-8.

43. Samloff IM, et al. Relationships among serum pepsinogen I, serum pepsinogen II, and gastric mucosal histology. A study in relatives of patients with pernicious anemia. Gastroenterology. 1982;83(1 Pt 2):204-9.

44. Ren JS, et al. Serum pepsinogens and risk of gastric and oesophageal cancers in the General Population Nutrition Intervention Trial cohort. Gut. 2009;58(5):636-42.

45. Di Mario F, et al. 'Serological biopsy' in first-degree relatives of patients with gastric cancer affected by Helicobacter pylori infection. Scand J Gastroenterol. 2003;38(12):1223-7.

46. Adamu MA, et al. Incidence and risk factors for the development of chronic atrophic gastritis: five year follow-up of a populationbased cohort study. Int J Cancer. 2011;128(7):1652-8.

47. Nishizawa T, et al. Family history is an independent risk factor for the progression of gastric atrophy among patients with Helicobacter pylori infection. United Eur Gastroenterol J. 2017;5(1):32-6.
48. Wang JT, et al. Direct DNA amplification and restriction pattern analysis of Helicobacter pylori in patients with duodenal ulcer and their families. J Infect Dis. 1993;168(6):1544-8.

49. Blecker U, et al. Evolution of Helicobacter pylori positivity in infants born from positive mothers. J Pediatr Gastroenterol Nutr. 1994;19(1):87-90.

50. Ashorn M, et al. Seroepidemiological study of Helicobacter pylori infection in infancy. Arch Dis Child Fetal Neonatal Ed. 1996;74(2):F141-2.

51. Gold BD, et al. Helicobacter pylori acquisition in infancy after decline of maternal passive immunity. Pediatr Res. 1997;41(5):641-6.

52. Murff HJ, Spigel DR, Syngal S. Does this patient have a family history of cancer? An evidence-based analysis of the accuracy of family cancer history. JAMA. 2004;292(12):1480-9.

53. Kleinbaum DG, Kupper LL, Morgenstern H. Epidemiologic research: principles and quantitative methods, vol. xix. Belmont: Lifetime Learning Publications; 1982. p. 529.

54. Oliveira C, et al. Familial gastric cancer: genetic susceptibility, pathology, and implications for management. Lancet Oncol. 2015;16(2):e60-70.

55. McLean MH, El-Omar EM. Genetics of gastric cancer. Nat Rev Gastroenterol Hepatol. 2014;11(11):664-74.

56. Sereno M, et al. Gastric tumours in hereditary cancer syndromes: clinical features, molecular biology and strategies for prevention. Clin Transl Oncol. 2011;13(9):599-610. 